\title{
Venous Thromboembolism During Treatment with Antipsychotics: A Review of Current Evidence
}

\author{
Anna K. Jönsson ${ }^{1,2} \cdot$ Johan Schill $^{3} \cdot$ Hans Olsson ${ }^{3}$ O Olav Spigset ${ }^{4,5}$ • \\ Staffan Hägg ${ }^{6}$
}

Published online: 8 February 2018

(C) The Author(s) 2018. This article is an open access publication

\begin{abstract}
This article summarises the current evidence on the risk of venous thromboembolism (VTE) with the use of antipsychotics. An increasing number of observational studies indicate an elevated risk of VTE in antipsychotic drug users. Although the use of certain antipsychotics has been associated with VTE, current data can neither conclusively verify differences in occurrence rates of VTE between first- and second-generation antipsychotics or between individual compounds, nor identify which antipsychotic drugs have the lowest risk of VTE. The biological mechanisms involved in the pathogenesis of this adverse drug reaction are still to be clarified but hypotheses such as drug-induced sedation, obesity, increased levels of antiphospholipid antibodies, enhanced platelet aggregation, hyperhomocysteinaemia and hyperprolactinaemia have been suggested. Risk factors associated with the underlying psychiatric disorder may at least partly explain the
\end{abstract}

Staffan Hägg

staffan.hagg@liu.se

1 Department of Drug Research, Section of Clinical Pharmacology, Department of Medical and Health Sciences, Linköping University, Linköping, Sweden

2 Department of Forensic Genetics and Forensic Chemistry, National Board of Forensic Medicine, Linköping, Sweden

3 Department of Psychiatry, Region Jönköping County, Department of Medical and Health Sciences, Linköping University, Linköping, Sweden

4 Department of Clinical Pharmacology, St. Olav University Hospital, Trondheim, Norway

5 Department of Clinical and Molecular Medicine, Norwegian University of Science and Technology, Trondheim, Norway

6 Futurum, Region Jönköping County, Department of Medical and Health Sciences, Linköping University, Sweden increased risk. Physicians should be aware of this potentially serious and even sometimes fatal adverse drug reaction and should consider discontinuing or switching the antipsychotic treatment in patients experiencing a VTE. Even though supporting evidence is limited, prophylactic antithrombotic treatment should be considered in risk situations for VTE.

\section{Key Points}

Increasing evidence shows an elevated risk of venous thromboembolism (VTE) in antipsychotic drug users.

In general, no well-documented differences in the occurrence rate of VTE between first- and secondgeneration antipsychotics or between individual antipsychotic drugs have been shown.

The biological mechanisms involved in the pathogenesis of antipsychotic-induced VTE are still largely unknown.

Physicians should be aware of this potentially serious adverse drug reaction and consider discontinuing or switching the antipsychotic treatment in patients experiencing VTE.

The threshold for considering prophylactic antithrombotic treatment should be low when risk situations for VTE arise in users of antipsychotics. 


\section{Introduction}

Venous thromboembolism (VTE) is a multicausal disease encompassing both deep vein thrombosis (DVT) and pulmonary embolism (PE) [1, 2]. VTE affects approximately $1.0-1.8$ per 1000 adults annually, may lead to complications such as post-thrombotic syndrome (PTS) and chronic thromboembolic pulmonary hypertension (CTPH), and can produce a considerable burden of morbidity and mortality $[1,2]$. The presence of one, or a combination of, components of Virchow's triad-hypercoagulability, stasis of blood flow and vein wall injury-are fundamental to developing VTE and all known risk factors for VTE are considered to affect at least one of these components [1-3]. Established risk factors are listed in Table 1 and include inherited coagulation abnormalities, malignancies, surgery, pregnancy, and medications such as oral contraceptives and hormone replacement therapy [1-4]. VTE has also been associated with psychiatric disorders and antipsychotic treatment [1-4].

In early, uncontrolled, observational studies [5-10], a relatively high incidence of PE among patients with schizophrenia and related disorders was reported but the association was not widely acknowledged. However, in the late 1990s, the association again received attention when an increased mortality rate in PE was reported in current users of clozapine compared with past users in a record linkage study on mortality in clozapine users [11, 12]. A few years later, this potential safety concern was strengthened by the publication of a case series of VTE in clozapine users [12], followed by a large nested casecontrol study [13] reporting a sevenfold increased risk of VTE in patients currently treated with first-generation antipsychotics (FGAs) relative to non-users. Since then, an increasing number of reports supporting and describing this association have been published. The available evidence on this link has previously been summarised by both us [14-16] and others [17-21], including two meta-analyses $[22,23]$. The aim of this article is to update and critically review the available data on incidence, risk factors, characteristics, mechanisms and management of VTE in users of antipsychotics.

In October 2017, the PubMed and Scopus databases were searched for relevant articles on antipsychotic medication and VTE. In the PubMed search, the Medical Subject Heading (MeSH) terms 'embolism and thrombosis', 'antipsychotic agents' and 'schizophrenia' were used,
Table 1 Major risk factors for VTE [1-4]

\begin{tabular}{lll}
\hline Clinical factors & Surgical factors & Inherited factors \\
\hline Advanced age & Central venous access & Antithrombin deficiency \\
Hospitalisation for acute medical illness & Major surgery & Dysfibrinogenemia \\
Long-haul flights (duration $>4 \mathrm{~h}$ ) & Orthopaedic surgery & Factor V Leiden mutation \\
Obesity & Trauma or fracture & Protein C deficiency \\
Pregnancy, including the post-partum period & & Protein S deficiency \\
& & Prothrombin 20210A mutation \\
\hline Medical diseases & Drugs & Mixed or unknown factors \\
\hline Antiphospholipid syndrome & Antiestrogens & Activated protein C resistance \\
Congestive heart failure & Antipsychotics & in the absence of factor V \\
Inflammatory bowel disease & Chemotherapy & Leiden mutation \\
Malignancy & Heparin-induced & Family history of VTE \\
Myeloproliferative disorders & thrombocytopenia & High levels of factor VIII \\
Myocardial infarction & High-dose therapy & Hyperhomocysteinaemia \\
Polycythemia vera & with progestogens & Lupus anticoagulant \\
Previous VTE & Hormone replacement & \\
Sepsis & therapy & \\
Stroke & Oral contraceptives & \\
Varicose veins & Vaginal ring for & \\
& contraception & \\
& Strontium ranelate & \\
& Thalidomide and & \\
\hline
\end{tabular}

VTE venous thromboembolism 
while in Scopus, the title, abstract and keywords were searched for a combination of one of the following terms: 'embolism', 'thrombosis' and 'thromboembolism' and one of the terms 'antipsychotic agents', 'antipsychotic agent', 'neuroleptic agent', 'neuroleptic agents', 'antipsychotic drug', 'antipsychotic drugs', or any one of 70 individual antipsychotic compounds. In addition, reference lists of identified relevant articles were scrutinised for additional studies not identified by the original database searches.

\section{Early Studies}

In the early 1950s, a few years after the discovery of chlorpromazine, some cases of fatal PE during treatment with antipsychotics were reported [24, 25]. Thereafter, case reports [26, 27], case series [5-10, 28-30] and observational studies [31, 32] describing VTE in FGA users were published, suggesting a link between VTE and psychosis; however, these studies were inconclusive as they lacked control groups or did not have detailed information about possible confounders. Thus, the association fell into oblivion until 1997 when it was again reported [11]. Thereafter, an increasing body of evidence has been reported in the literature supporting a relationship between VTE and antipsychotics. The current systematic observational data for the risk of VTE among users of antipsychotics are summarised in Table 2 (case-control studies) and Table 3 (cohort studies and drug surveillance studies).

\section{Case-Control Studies}

\subsection{General Patient Populations}

Most of the systematic studies investigating the association between VTE and antipsychotics are case-control studies in adult patients. The first of these studies was a nested case-control study published in the year 2000 using data from the UK General Practice Research Database [13]. This study found a 7.1-fold increased risk of VTE in current antipsychotic users younger than 60 years of age compared with matched control subjects without such use. The highest risk was noted in the first 3 months of treatment. There was a trend that low-potency FGA users had a higher VTE risk than high-potency FGA users, but the confidence intervals (CIs) were wide and overlapping. As only a few second-generation antipsychotic (SGA) users were included in this study, no conclusive results regarding this association could be presented. The following year, a subgroup analysis of data from a case-control study [57] in Dutch outpatients (the Leiden Thrombophilia Study) was published, reporting a twofold increased risk of VTE in outpatients who used unspecified antipsychotic drugs compared with control subjects; however, due to the limited number of study subjects, the CIs were wide and the results were consequently inconclusive.

In a French hospital-based case-control study [33] designed to evaluate interactions between acquired and inherited risk factors of VTE, the use of antipsychotics was associated with a 3.5-fold increased risk of VTE. Drug exposure was defined as current use of drugs at hospital admission. In a retrospective case-control study in adult hospitalised patients at a Czech university hospital [34], the use of antipsychotic drugs was 2.8-fold more common in patients who had been hospitalised for VTE compared with the control subjects with hypertension treated at the same hospital. In a Danish nested case-control study [35] a 2.0fold increased risk of VTE was found in current users of antipsychotic drugs compared with non-users. Former users of antipsychotic drugs had a non-significant elevated risk of VTE compared with non-users. Even though a wide range of confounding factors were controlled for in the analyses, smoking, diet, obesity and schizophrenic behaviour could not be adjusted for, which may have affected the results. As it was not possible to separate the risk of VTE between current and former users of antipsychotics, it is possible that lifestyle factors, the underlying disease or residual confounding could, at least to some extent, explain the increased risk.

In a large, UK, population-based, case-control study [36] using data from the QResearch primary care database, those who had used antipsychotic drugs in the previous 24 months had a 1.3-fold greater risk of VTE than non-users. Compared with control subjects, 2.0-fold and 1.7-fold increased risks were observed in users who had started their antipsychotic treatment in the previous 3 months and in SGA users, respectively. In addition, there was a nonsignificant tendency toward a higher risk in low-potency FGA users than in high-potency FGA users. Statistically significant increased risks were observed for quetiapine, haloperidol, chlorpromazine, olanzapine and prochlorperazine, but not for risperidone or trifluoperazine; however, the latter non-significant results may be due to the limited number of exposed individuals. The number of extra cases of VTE per 10,000 patients treated with antipsychotics over 1 year was estimated to be four in patients of all ages and ten in patients aged 65 years and older. In another nested case-control study [37] based on data from a UK primary care database (Clinical Practice Research Datalink), no significant increased risk of VTE was found in users of any antipsychotic drugs compared with non-users; however, for users who had received a new prescription less than 31 days ago, a 3.2-fold increased risk was found. Prochlorperazine was associated with an increased risk, but not risperidone, olanzapine, haloperidol or chlorpromazine. 


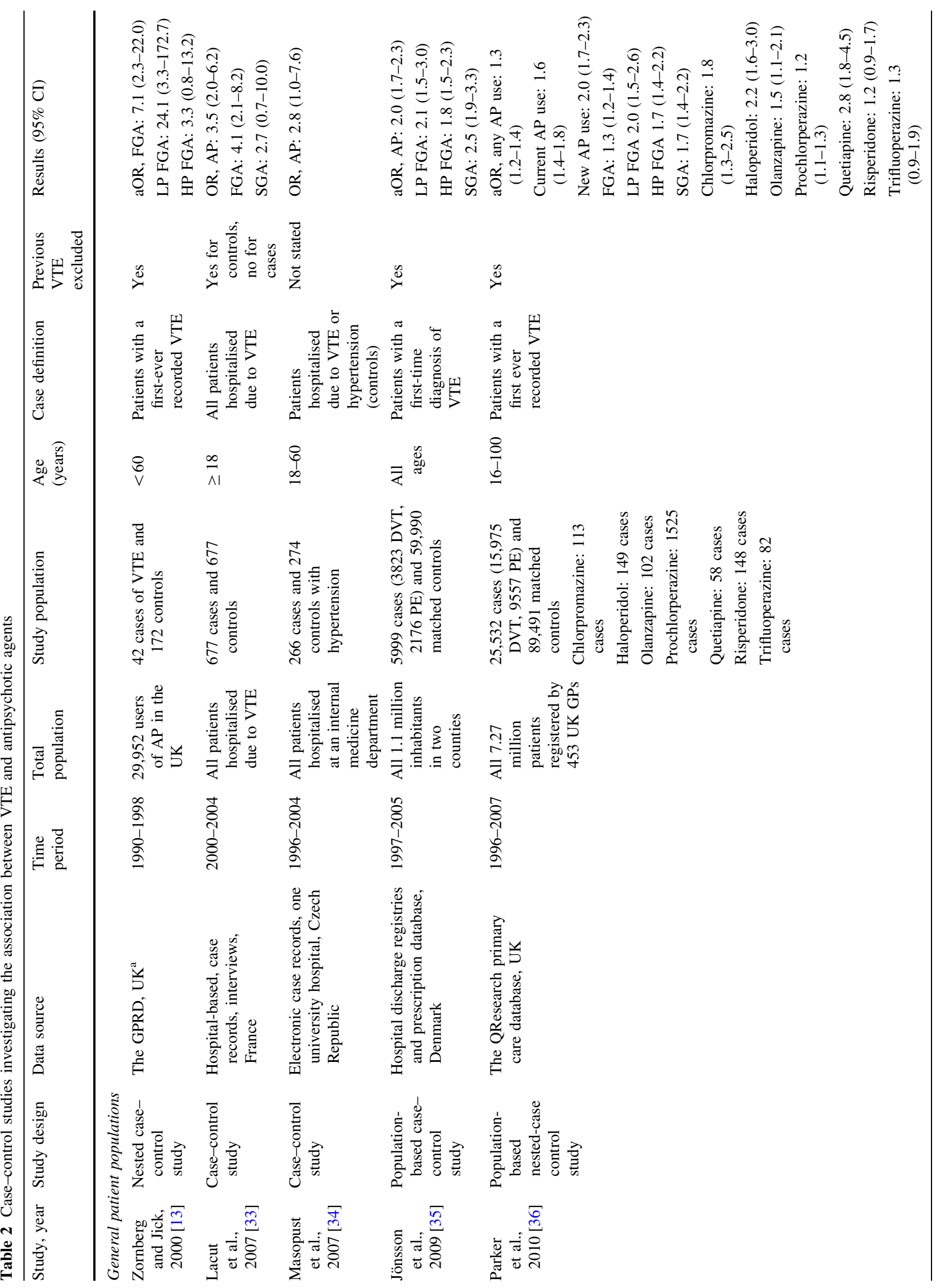




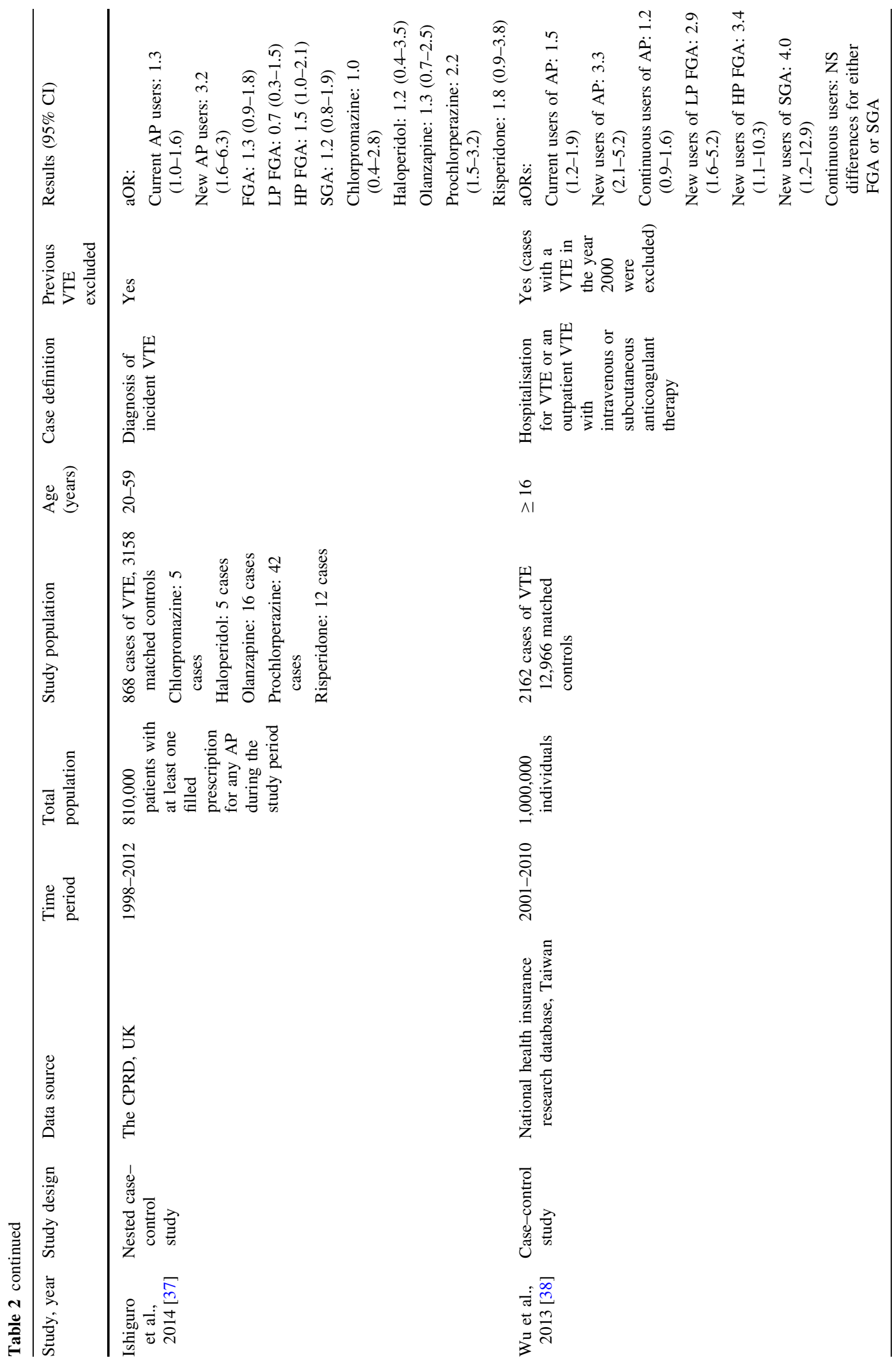




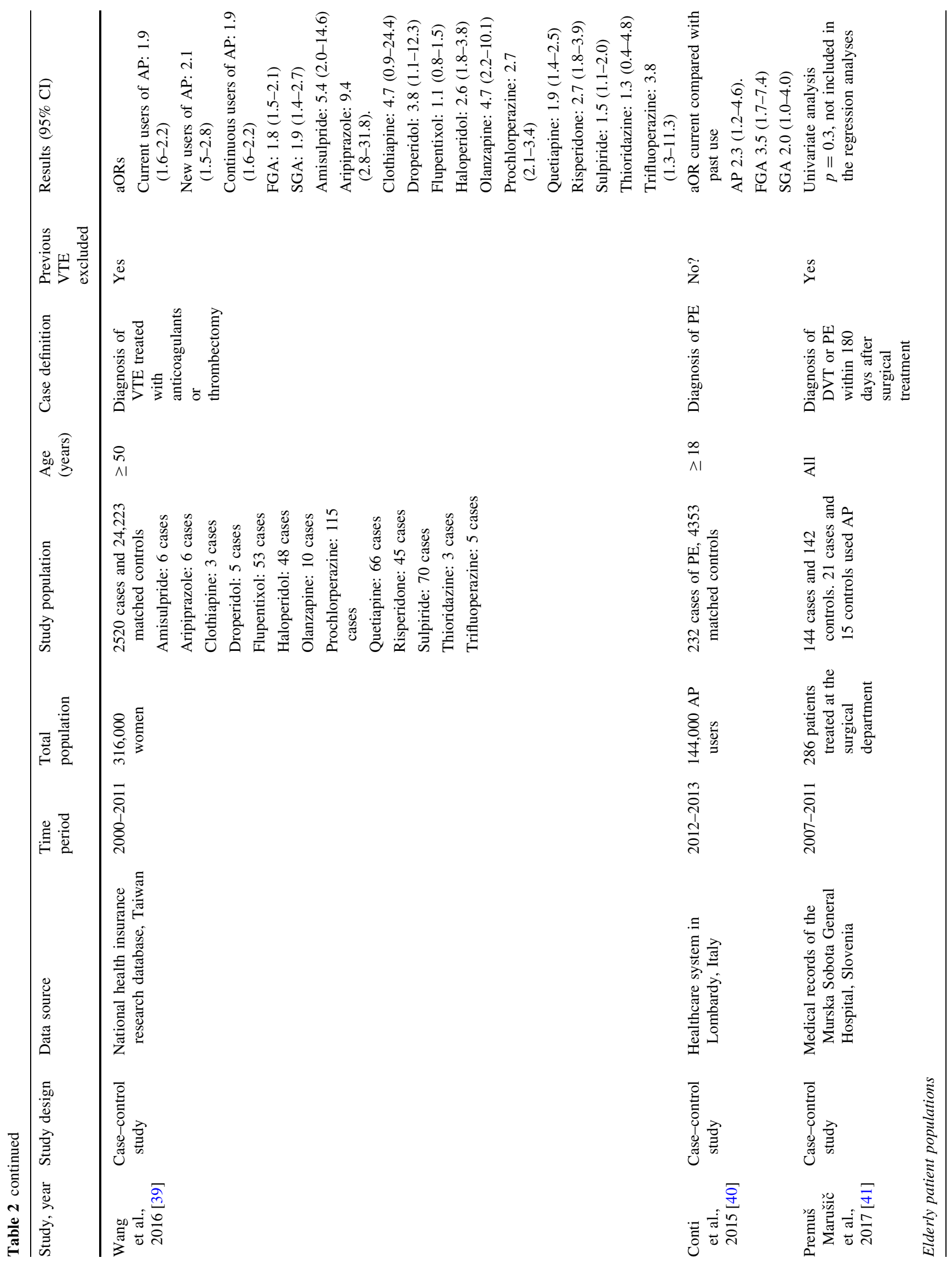




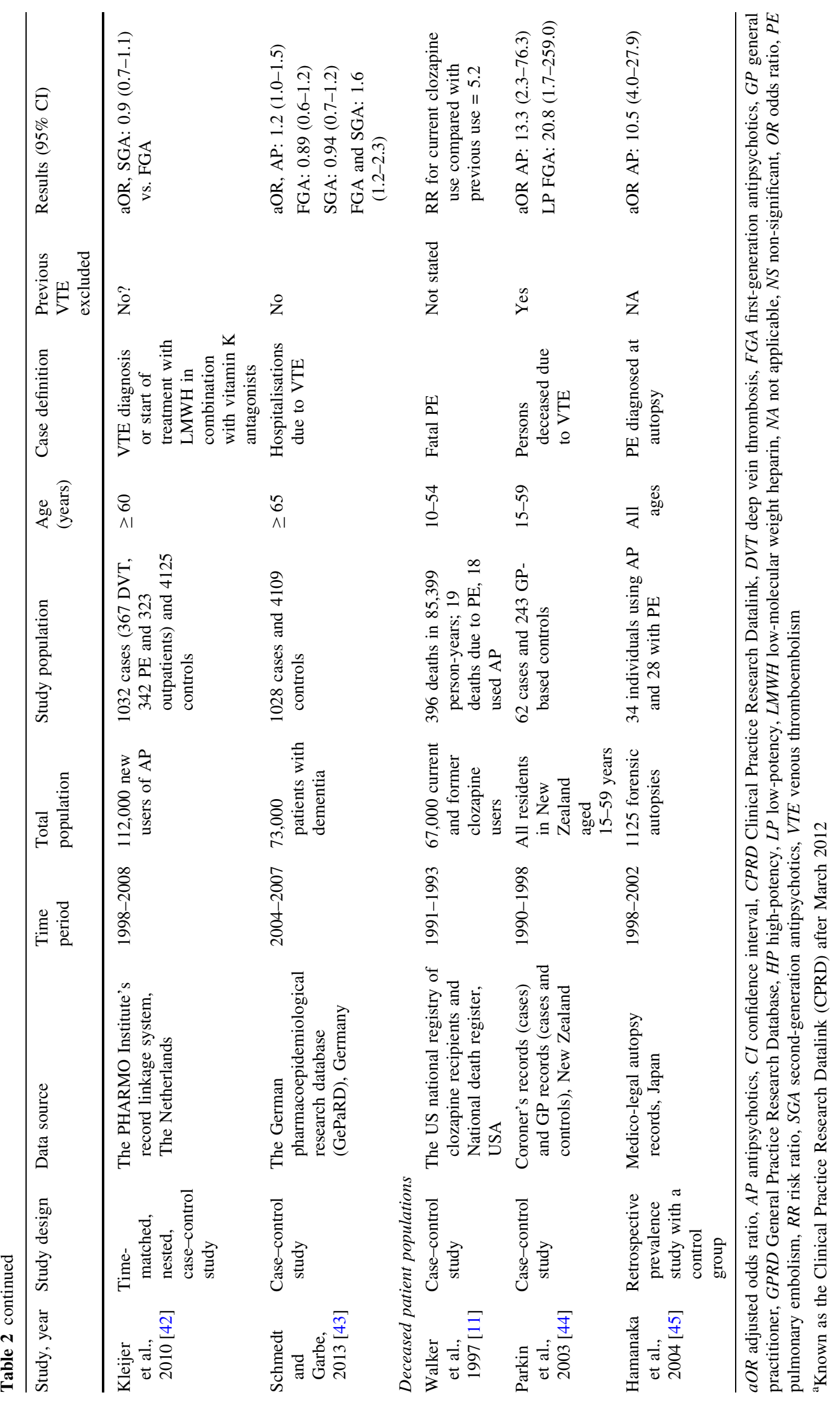




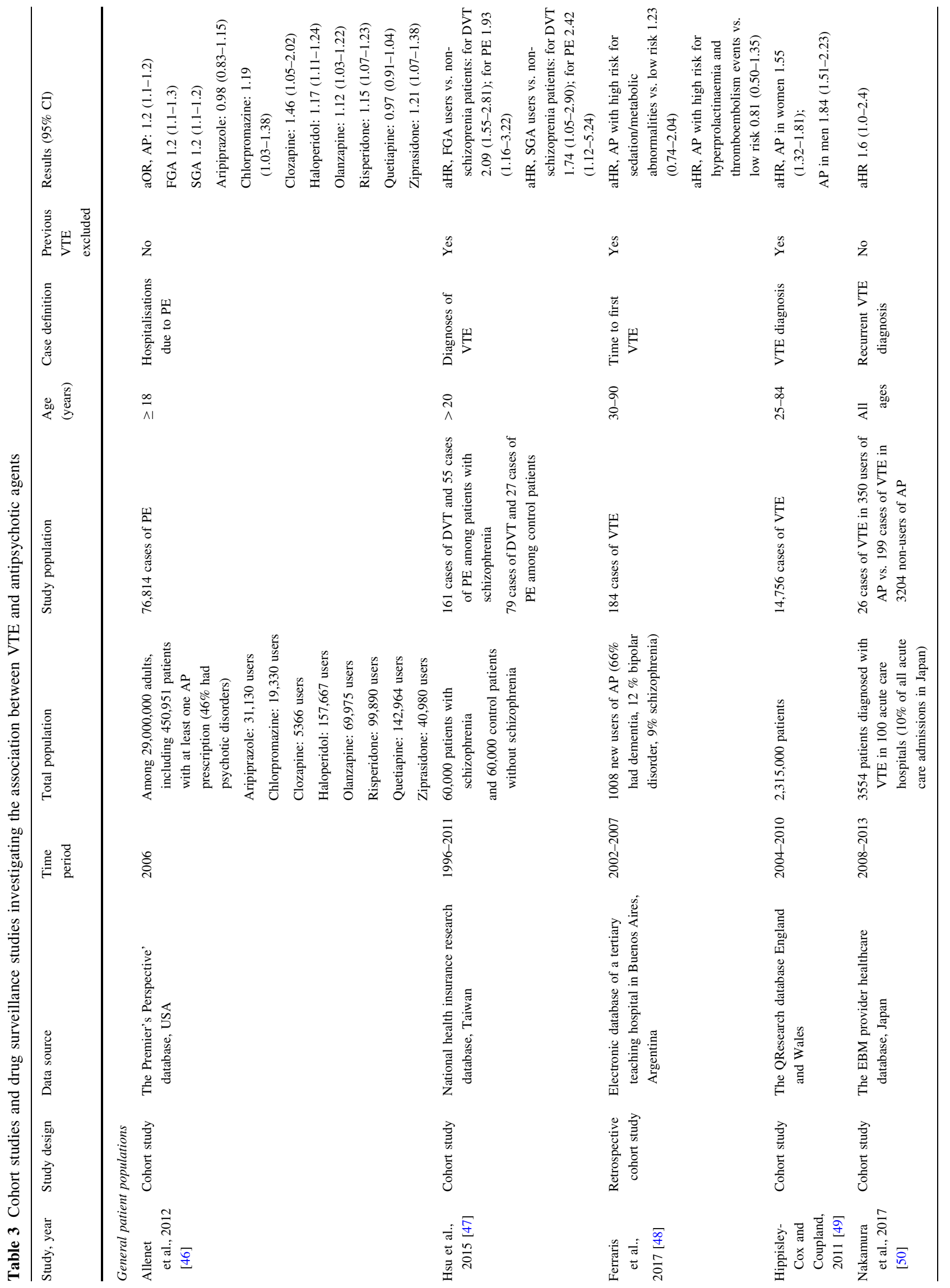




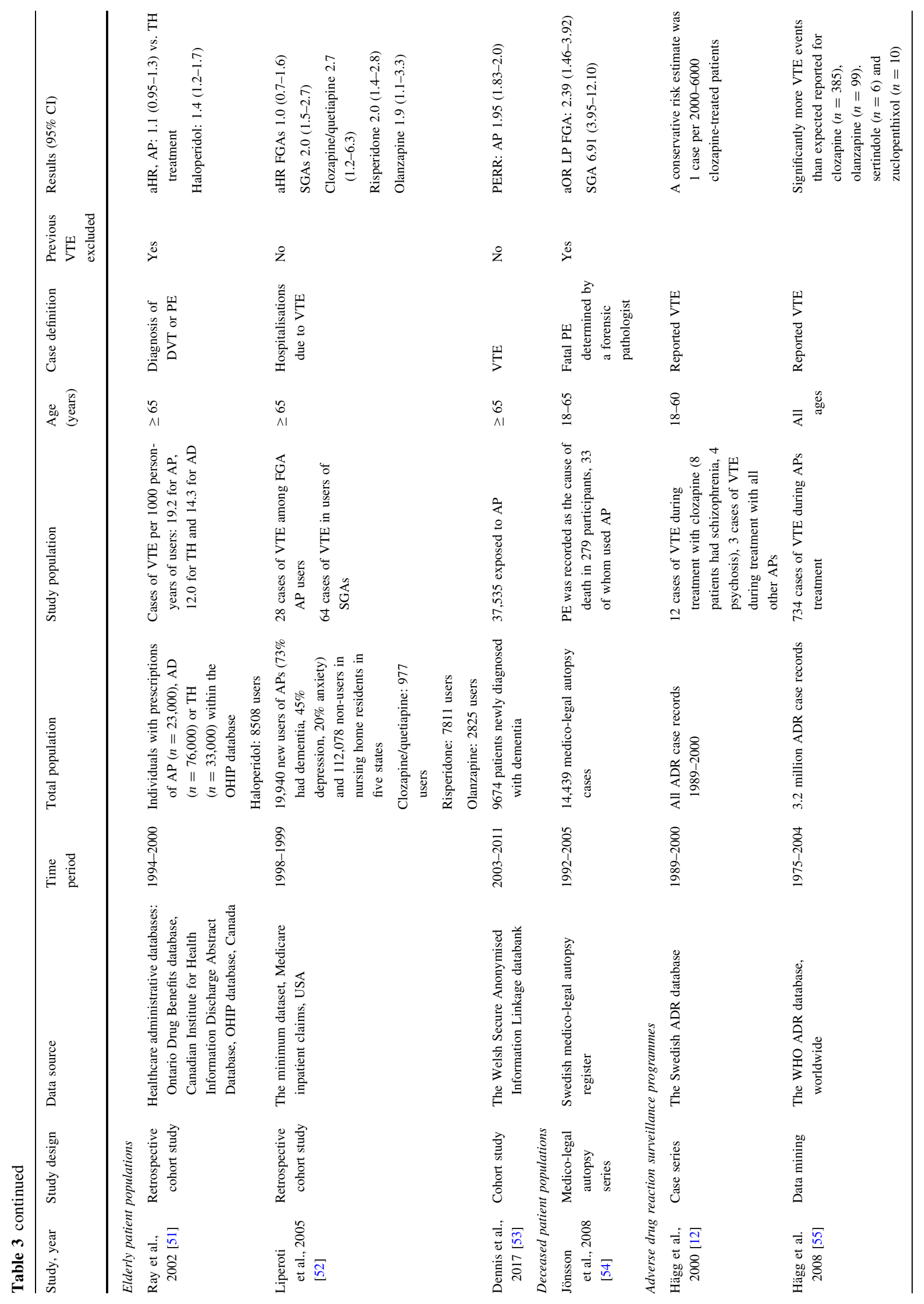




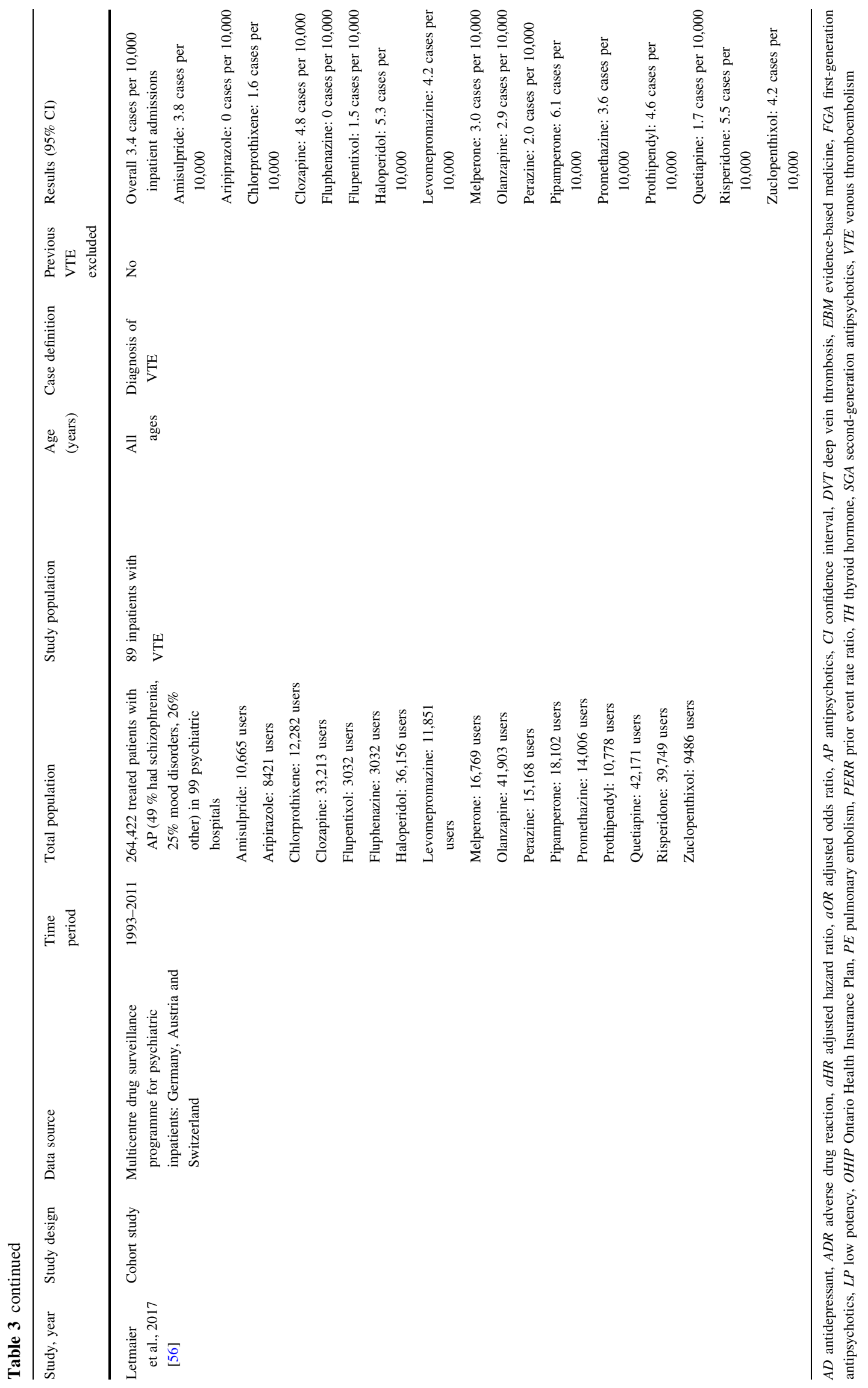


However, the ability to examine the risks of individual substances was limited by the relatively small number of cases.

Two case-control studies have used data from the National Health Insurance Research Database in Taiwan. In the first study [38], current users of antipsychotics were found to have a 1.5-fold increased risk and new users had a 3.3-fold increased risk of VTE compared with matched controls. In continuous antipsychotic users, no significant increased risk could be observed. In the second study [39], only women older than 50 years of age were included. In that study, current antipsychotic users had a 1.9-fold increased risk and new users had a 2.1-fold increased risk. Among current users of antipsychotics, the risk was similar among FGA and SGA users. An increase of VTE was observed in users of the following individual antipsychotics: aripiprazole, amisulpride, olanzapine, trifluoperazine, droperidol, prochlorperazine, risperidone, haloperidol, quetiapine, and sulpiride. In contrast, no increased risk was found for flupentixol, clothiapine and thioridazine, which might be explained by the low number of users.

In a large Italian study [40] investigating the relationship between antipsychotics and PE, current use of antipsychotics was associated with a statically significant 2.3 -fold increased risk of PE compared with past use. The increased risk could be observed in both FGA and SGA users. In a small Slovenian retrospective case-control study [41] of surgical patients at a single hospital, no significant difference in the proportions of subjects using antipsychotics between VTE cases and control subjects was found.

\subsection{Elderly Patient Populations}

In a large, time-matched, nested, case-control study [42], data from the Dutch PHARMO Institute database was analysed within a cohort of patients aged 60 years and above. Current exposure to antipsychotic drugs was not associated with an increased risk of VTE compared with non-use, and no statistically significant associations between dosage, duration of use, or type of antipsychotic drug and the risk of VTE were found. In another casecontrol study [43], the association between the use of antipsychotics and the risk of VTE in elderly patients with dementia was investigated using data from the German Pharmacoepidemiological Research Database. A modest but statistically significant 1.2 -fold increased risk was found in current antipsychotic users and a 1.6-fold increased risk was observed in users of FGA and SGA in combination. In current users, only recent commencement was associated with an increased risk. There were no significantly increased risks of VTE when SGA and FGA were analysed separately.

\subsection{Deceased Patient Populations}

In a clozapine mortality study [11], data from the US national registry of clozapine recipients were linked to the National Death Register. In this study, current clozapine users had a fivefold increased mortality rate in PE compared with previous users. Selective factors leading to an increased autopsy rate in psychiatric patients cannot be excluded, but the referral rate of such patients to autopsy was low. In a nationwide New Zealand case-control study [44], a 13.3-fold increase in the risk of fatal PE was observed in current users of antipsychotic drugs compared with non-users. Low-potency FGA users had the highest risk-a 20.8-fold increased risk compared with control subjects. Thioridazine was the main drug in that study. In a Japanese study among cases of sudden unexpected death [45], a 10.5-fold increased risk of fatal PE was observed in antipsychotic drug users.

\section{Cohort Studies}

\subsection{General Patient Populations}

In a retrospective cohort study [46] of a US hospital database consisting of 450,000 users of antipsychotics, a modest but statistically significant 1.2 -fold increased risk of PE was observed compared with non-users. FGA and SGA users had comparable risks for PE. In the dataset, SGA represented over $78 \%$ of the prescriptions, but haloperidol was the most common individual drug. Clozapine users had the highest risk, and marginally increased risks were noted for users of ziprasidone, chlorpromazine, haloperidol, risperidone, and olanzapine. Quetiapine and aripiprazole users had no increased risk. The risk of PE increased with higher doses.

In a Taiwanese population-based cohort study [47] claims data from the National Health Insurance Research Database were used. Patients with schizophrenia using FGA had hazard ratios of 2.1 and 1.9 for DVT and PE, respectively, and patients with schizophrenia using SGA had hazard ratios of 1.7 and 2.4 for DVT and PE, respectively, compared with non-schizophrenia patients. There were no significant differences between FGA and SGA users. In an Argentinian retrospective cohort study [48], patients initiating antipsychotic therapy were followed for 36 months. The incidence rate was 18.4 cases per 10,000 person-years. SGA and FGA were divided into subgroups based on their tendency to cause sedation, metabolic abnormalities and hyperprolactinaemia, and there were no significant differences in VTE risk between any of these subgroups. 
In a large, prospective, open cohort study using routinely collected data from general practices in the UK (the QResearch Database), a new clinical risk prediction algorithm was developed and validated [49]. In this study, 1.8and 1.6-fold increased risks of VTE were found for male and female antipsychotic users, respectively. In a cohort study [50] undertaken in a Japanese acute-care hospital, the rate of recurrence of VTE events was 7.2 per 100 patientyears in patients with a previous VTE. Three predictors for recurrent VTE were identified: malignant disease, antipsychotic drug use and non-steroidal anti-inflammatory drug (NSAID) use.

\subsection{Elderly Patient Populations}

Three cohort studies have investigated the association between VTE and antipsychotic medication in the elderly. In a Canadian retrospective cohort study in subjects aged $\geq 65$ years [51], no increase was observed in the rate of hospitalisation for VTE among users of antipsychotic drugs compared with users of thyroid hormones, although a subgroup analysis showed a modest increased risk of VTE among users of haloperidol. In a retrospective cohort study of US nursing home residents aged $\geq 65$ years [52], no statistically significant increased rate of hospitalisation for VTE in FGA users relative to non-users of antipsychotics was found; however, in SGA users a statistically significant twofold increased rate was found. Statistically significant increased risks were observed in users of clozapine or quetiapine, users of risperidone and users of olanzapine. In a retrospective study [53] of a population-based cohort of patients with newly diagnosed dementia in the Welsh Secure Anonymised Information Linkage databank, a twofold increased risk of a venous thromboembolic episode was found in antipsychotic users.

\subsection{Deceased Patient Populations}

In an autopsy series [54] of deceased persons aged 18-65 years where PE was the cause of death, the presence of antipsychotic drugs in post-mortem blood analyses was investigated. A 2.4- and 6.9-fold increased risk of fatal PE was found in low-potency FGA and SGA users, respectively. No increased risk was found in high-potency FGA users but very few individuals were included in this group.

\subsection{Adverse Drug Reaction Surveillance Programmes}

In an analysis of the Swedish national pharmacovigilance register from the year 2000, 12 cases of VTE during clozapine treatment were identified [12]. In five cases, the outcome was fatal. Based on these cases, as well as national sales statistics, it was estimated that at least one per 2000-6000 treated patients experienced a VTE. Similar numbers for clozapine as well as for other antipsychotics were reported from Germany and Switzerland the same year [58]. In a study of the WHO international database of adverse drug reactions from 2008, a total of 754 suspected cases of VTE related to antipsychotics were reported [55]. After applying a data mining technique on this database, a robust association between VTE and SGA was found, but not with high- or low-potency FGA. Occurrence of VTE was more frequently reported during treatment with clozapine, olanzapine, sertindole and zuclopenthixol than during treatment with other antipsychotics. In a systematic surveillance programme of severe adverse drug reactions in 99 psychiatric hospitals in Germany, Switzerland and Austria, 3.4 cases of VTE per 10,000 inpatient admissions, corresponding to 43 cases per 10,000 person years, were identified in antipsychotic users [56]. Of these subjects, $73 \%$ had another risk factor for VTE, most often physical restraint. There was no difference between the low-potency FGA users, high-potency FGA users and SGA users. The highest occurrence was found in users of pipamperone, with 6.1 cases per 10,000 inpatient admissions, followed by risperidone (5.5 cases), haloperidol (5.3 cases), clozapine (4.8 cases), and prothipendyl (4.6 cases).

\section{Meta-Analyses}

A meta-analysis of seven case-control studies [22], involving 31,095 cases and 143,472 controls, was published in 2011. In this analysis, a 2.4-fold overall increased risk (95\% CI 1.7-3.4) of VTE was reported in users of antipsychotics. Although the reported risks were higher in SGA users (odds ratio [OR] 2.20, 95\% CI 1.22-3.96) than in FGA users (OR 1.72, 95\% CI 1.31-2.24) and higher in low-potency FGA users (OR 2.91, 95\% CI 1.80-4.71) than in high-potency FGA users (OR 1.58, 95\% CI 1.50-1.67), the wide and overlapping CIs, uncertainties regarding drug classification, and the heterogeneity in methodologies used, make the observed differences difficult to interpret.

In a meta-analysis of 17 case-control and cohort studies published in 2014 [23], antipsychotic use was associated with a 1.5-fold increased risk of VTE (95\% CI 1.28-1.86). Increased VTE risks were revealed in FGA users (OR 1.74, 95\% CI 1.28-2.37; six studies) and SGA users (OR 2.07, 95\% CI 1.74-2.52; five studies). No significantly increased risk of $\mathrm{PE}$ could be verified in antipsychotic users (OR 4.90, 95\% CI 0.77-30.98; three studies). However, after adding the results of a recent case-control study [40], an updated meta-analysis could reveal a statistically increased risk of $\mathrm{PE}$ (OR 3.68, 95\% CI 1.22-11.07; four studies) [40]. 


\section{Interpretation of Observational Studies}

Despite different methodologies, study populations and antipsychotic drug use patterns, most observational studies demonstrate an increased risk for VTE in users of antipsychotics. As case-control studies in general are known to overestimate risks, additional cohort studies are therefore important to verify and confirm results from case-control studies. Regarding the link between VTE and antipsychotics, cohort studies have reported similar findings as case-control studies, and there is also a consistency in overall results across different studies.

The highest VTE risk can be observed in present users of antipsychotics [36-39], especially the first 3 months of treatment [12, 36-39], suggesting that the medication rather than the underlying disease is the most important factor. Based on available data, it is complicated to determine which antipsychotic drug type carries the highest risk of VTE. In the meta-analysis from 2011 [22], pooled estimates showed that low-potency FGA had the highest risk, followed by SGA, but with overlapping CIs. In the meta-analysis published in 2014 [23], the risk of VTE was slightly higher in SGA users than in FGA users, but, again, CIs were overlapping. In these two meta-analyses, only a few of the included studies provided data on individual drugs, and pooled estimates of the VTE risk were therefore uncertain. More recently, a few larger studies $[36,39,46,52]$ have been able to study the risk of VTE related to single compounds, but current data can neither conclusively verify the existence of a difference between individual compounds nor identify the antipsychotic drug(s) having the lowest risk of VTE. Therefore, additional, even larger studies should be carried out, especially prospective cohort studies having valid information on already known risk factors for VTE, thereby being able to control for all potentially confounding factors.

\section{Biological Mechanism}

The biological mechanisms responsible for the observed increased risk of VTE in antipsychotic drug users are not known and no single biological mechanism is likely to explain this adverse reaction. Several hypotheses have been put forward and proposed mechanisms are related to factors of the antipsychotic drug, the underlying disease, or a combination of these.

Drug-related factors include the frequently observed adverse drug reactions sedation, weight gain and hyperprolactinaemia. Sedation and weight gain pave the way to immobilisation and venous stasis in the lower extremities, thus increasing the risk of VTE $[59,60]$. Prolactin is a potent platelet aggregation co-activator and the release of this hormone is inhibited by continuous dopamine release in the pituitary $[61,62]$. This effect is mediated via dopamine $\mathrm{D}_{2}$ receptors. As antipsychotics are $\mathrm{D}_{2}$ receptor antagonists [63], prolactin increase is a prominent adverse effect, particularly for high-potency FGAs and risperidone. Aripiprazole and clozapine, which show partial agonistic activity and low affinity for dopamine $\mathrm{D}_{2}$ receptors, respectively, show less or no prolactin increase [64]. Nonetheless, also clozapine is associated with an increased risk of VTE. Conflicting evidence regarding platelet adhesion and aggregation in vitro for clozapine and other antipsychotics make the clinical impact of these findings unclear $[14,65,66]$; it should also be noted that these platelet effects would be expected to cause arterial rather than venous thromboembolic events.

Antiphospholipid antibodies (APLs), including anticardiolipin antibodies and lupus anticoagulants, have been shown to be increased in patients using FGA and clozapine [67-76]. An enhanced level of APLs is an established risk factor for VTE. However, the clinical impact of a high level of APLs is uncertain since a high level has also been shown in patients not using antipsychotics and only few cases of VTE in patients treated with antipsychotics have been associated with high levels of APLs [73, 75, 77]. Two more recent studies did not find any association between antipsychotic treatment and APL levels [78, 79].

A few well-established risk factors for VTE (Table 1), such as obesity and sedentary lifestyle, are abundant in people with psychotic disorders. Besides being a risk factor by its own, obesity increases thrombin formation and decreases fibrinolytic activity [4]. Obesity is also linked to myocardial infarction and congestive heart failure, conditions associated with VTE. Patients with psychoses clearly have a more sedentary lifestyle than age- and sex-matched controls from the general population, and their often low socioeconomic status and income may reduce the practical possibilities to make healthy lifestyle choices [80].

In the acute phase of a psychotic illness, physical restraint due to uncontrollable behaviour may also increase the risk of VTE. In a case series [81], 22 patients with PE related to catatonic syndrome were described, suggesting that certain psychiatric symptoms may have an increased risk of VTE. Of women admitted to a US hospital, two of four women with the catatonic type of schizophrenia developed VTE, compared with none of 168 women not having this type of schizophrenia [82]. The incidence of VTE was investigated in a Japanese study by means of routine Doppler ultrasound scanning in 79 catatonic patients who were involuntarily hospitalised and 292 noncatatonic physically restrained patients [83]. The incidence of VTE was significantly higher in the catatonic patients (25\%) compared with the restrained non-catatonic patients (11\%). 
Table 4 Suggested algorithm of primary prevention of VTE for hospitalised psychiatric patients [18]

\begin{tabular}{|c|c|}
\hline Score 2 risk factors & Score 1 risk factors \\
\hline $\begin{array}{l}\text { History of deep vein thrombosis or pulmonary embolism } \\
\text { Malignancy (active/treated) } \\
\text { Age } \geq 75 \text { years } \\
\text { Acute infection (including severe infection/sepsis) or acute respiratory disease } \\
\text { (including exacerbation of chronic respiratory disease) }\end{array}$ & 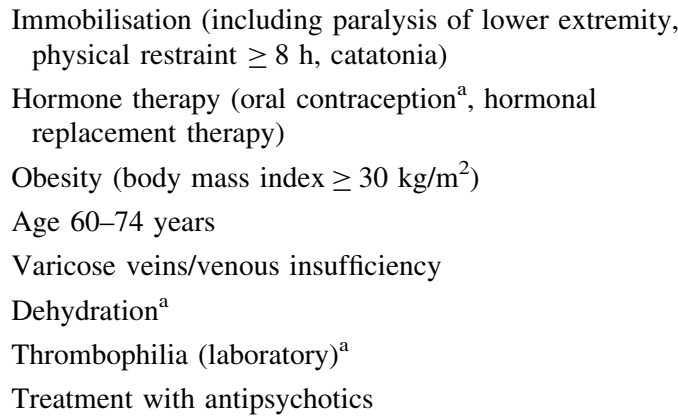 \\
\hline
\end{tabular}

The VTE risk was classified as low at a score of 0-3, medium at a score of 4-7, and high at a score of $\geq 8$. Prophylaxis is recommended when the score is $\geq 4$

$V T E$ venous thromboembolism

${ }^{a}$ In the alternative VTE risk factor scoring system for psychogeriatric inpatients by Liu and colleagues [98], oral contraceptive use and thrombophilia (laboratory) was removed. The presence of acute renal failure was added as a proxy for dehydration

High-sensitive C-reactive protein (CRP) and other inflammatory markers are increased in a subgroup of patients with acute psychosis [84], and inflammation parameters have been shown to increase initially when starting treatment with clozapine and haloperidol $[85,86]$; however, an increase in inflammatory response would be expected to cause arterial rather than venous thromboembolic events, rendering the clinical importance of this observation uncertain. In addition, markers of thrombogenesis, e.g. platelet activation (P-selectin) and coagulation (D-dimer and factor VIII), are triggered in acute psychosis, suggesting the disease itself may increase the risk of VTE [87]. Increased homocysteine levels have been under debate and have been proposed to increase the risk of VTE, and hyperhomocysteinaemia has been observed in patients with schizophrenia, as well as with the metabolic syndrome, which is an established adverse reaction of antipsychotic drug treatment [88, 89]. However, lifestyle factors such as a poor diet, cigarette smoking and high coffee and tea consumption may cause the observed increase in homocysteine seen in the group, rather than the antipsychotic drug or the disease itself [90].

In conclusion, although several mechanisms have been proposed to date, no clear-cut causal relationships between the mechanisms of action or other biological effects of antipsychotic drugs and VTE have been established.

\section{Clinical Implications and Management}

Due to the increased risk of thromboembolic events in users of antipsychotic medications, individual risk factors for VTE should be assessed and high-risk situations should be identified. The importance of identifying early signs of VTE in antipsychotic users is stressed by the relatively high risks for fatal VTE and PE in antipsychotic users $[44,45]$ compared with the general increased risk of VTE in antipsychotic users (Tables 2,3). Moreover, as the risk is dynamic, the risk should be re-evaluated when clinical circumstances change; for example, in situations with reduced mobility, infections, surgery and hospitalisation. In high-risk situations, appropriate prevention measures should be considered, i.e. reducing modifiable risk factors and commencing prophylactic antithrombotic treatment in line with guidelines [91]. To improve adherence to relevant guidelines, interventions such as medical education [92], computer-based alerts [93], and didactic lectures [94] have been suggested. Risk-assessment models (RAMs) have been advocated as instruments to ensure objective risk assessments and to support evidence-based clinical decisions in management of the risk [95, 96]. However, risk predictors differ across RAMs and several of them do not take antipsychotic treatment as a risk factor into consideration, possibly because limited evidence exists regarding how the increased risk of VTE due to antipsychotic agents should be handled in clinical practice.

Attempts to compile algorithms for the primary prevention of VTE for psychiatric inpatients have been made (Table 4). In the algorithm by Malý et al. [18], which is based on an algorithm by Cohen et al. [97], the patients' risk of VTE is assessed and preventive measures suggested. Prophylactic treatment with low-molecular weight heparin is suggested in hospitalised patients with reduced mobility until they are fully mobilised. Liu et al. [98] adopted the scoring system from Malý et al. [18] to assess the VTE risk in psychogeriatric inpatients. Here, oral contraceptive use 
Box 1 Clinical recommendations for the prevention and management of venous thromboembolism (VTE) in users of antipsychotics

Patients with risk factors for VTE besides the antipsychotic treatment should be informed of VTE as a possible adverse effect, early symptoms of VTE, and the importance of seeking medical care immediately if a VTE is suspected

Early symptoms of VTE should be recognised and further investigated without delay by healthcare providers

Prevention of VTE with low-molecular weight heparin is generally considered safe and effective and should be considered in antipsychotic users restrained for more than $24 \mathrm{~h}$ due to psychotic uncontrollable behaviour

The threshold for initiating prophylactic treatment with low-molecular weight heparins should be low in other VTE high-risk situations (for example, fractures, surgery and reduced mobility)

A manifest VTE should be treated according to current VTE management guidelines

After a diagnosis of VTE has been made in an antipsychotic drug user, the indication of the antipsychotic treatment should be re-evaluated and treatment suspended whenever possible, or, alternatively, switched to another antipsychotic compound

was removed, as was laboratory thrombophilia, which would rarely be checked in an elderly patient. The presence of acute renal failure was added as a proxy for dehydration. Patients were classified as having low (score 0-3), medium (4-7) and high risk ( $\geq 8)$, as in the scoring system by Malý et al. [18]. Those assessed to be at medium risk had a significantly increased rate of VTE and therefore the authors proposed prophylaxis to be prescribed for psychogeriatric inpatients assessed to be at medium and high risk. Nevertheless, the threshold for prophylactic treatment should also be low in situations with VTE risk factors that are not included in the algorithm. If no contraindications to low-molecular weight heparins are present, the prevention of VTE with such drugs is generally considered safe and effective. No data exist on the use of direct oral anticoagulants (DOACs), such as dabigatran, rivaroxaban, apixaban and edoxaban, in this situation. In patients not suitable for treatment with low-molecular weight heparins, DOACs could be considered as an alternative. In such cases, it seems reasonable to use these drugs in low doses, e.g. the doses approved for VTE prophylaxis after hip or knee replacement surgery.

High-risk individuals using antipsychotics should be informed of the symptoms of DVT and PE, as well as the importance of seeking medical care immediately. A manifest VTE or PE in a patient using an antipsychotic agent should be managed in accordance with current guidelines [91]. After diagnosis, the indication of the antipsychotic treatment should be re-evaluated and treatment suspended if possible. Both Malý et al. [18] and Liu et al. [98] recommend that if there is a strong indication for treatment, switching to an antipsychotic with a possibly lower association with VTE should be considered; however, they do not specify which antipsychotics should be preferred (or avoided) in patients with a high risk of VTE. Although solid evidence is lacking, it could be speculated that the risk of VTE might be higher for clozapine than for other SGAs [46, 52]. Separating the other SGAs regarding the risk of VTE is not possible based on available data. As psychotic disorders as such have been associated with VTE, it is possible that the underlying condition may have contributed in the etiology of VTE. When choosing an antipsychotic drug for a patient, it seems reasonable to consider not only drug-specific risk factors for VTE but also patient-specific risk factors. Clinical recommendations for the prevention and management of VTE in users of antipsychotics are summarised in Box 1.

\section{Conclusions}

Increasing evidence points towards an increased risk of VTE in patients using antipsychotics. The association is mainly supported on observational data. Even though individual studies have a number of methodological shortcomings, the overall picture suggests that the observed higher risk is related to the antipsychotic medications as such. The risk seems to be highest during the first 3 months of treatment. The currently available literature does not document any clear-cut differences in the risk of VTE between FGA and SGA or between individual antipsychotic drugs, and the biological mechanisms underlying the increased risk are still largely unknown.

In patients developing VTE, adequate antithrombotic treatment should be started and consideration should be given to discontinuing or switching the antipsychotic treatment. Even though solid evidence is lacking, it might be pertinent to avoid clozapine if possible, but giving advice regarding which drug(s) to prefer or avoid among the other SGAs is not possible. Prophylactic antithrombotic treatment should be given liberally to patients concomitantly having other risk factors of developing VTE, e.g. in line with the algorithm outlined in Table 4.

Funding No sources of funding were used in the preparation of this article. The open access fee was covered by Linköping University.

\section{Compliance with ethical standards}

Conflict of interest Anna K Jönsson, Johan Schill, Hans Olsson, Olav Spigset and Staffan Hägg have no conflicts of interest relevant to the content of this article. 
Open Access This article is distributed under the terms of the Creative Commons Attribution-NonCommercial 4.0 International License (http://creativecommons.org/licenses/by-nc/4.0/), which permits any noncommercial use, distribution, and reproduction in any medium, provided you give appropriate credit to the original author(s) and the source, provide a link to the Creative Commons license, and indicate if changes were made.

\section{References}

1. Heit JA, Spencer FA, White RH. The epidemiology of venous thromboembolism. J Thromb Thrombolysis. 2016;41:3-14.

2. Goldhaber SZ, Bounameaux H. Pulmonary embolism and deep vein thrombosis. Lancet. 2012;379:1835-46.

3. Crous-Bou M, Harrington LB, Kabrhel C. Environmental and genetic risk factors associated with venous thromboembolism. Semin Thromb Hemost. 2016;42:808-20.

4. Ageno W, Squizzato A, Garcia D, Imberti D. Epidemiology and risk factors of venous thromboembolism. Semin Thromb Hemost. 2006:32:651-8.

5. Maurice H. Des complications phlébitiques au cours des traitements par les neuroleptiques. L'Encephale. 1956;45:797-801.

6. Grahmann H, Suchenwirth R. Thrombosis hazard in chlorpromazine and reserpine therapy of endogenous psychoses [in German]. Nervenarzt. 1959;30:224-5.

7. Haefner $\mathrm{H}$, Brehm I. Thromboembolic complications in neuroleptic treatment. Compr Psychiatry. 1965;6:25-34.

8. Meier-Ewert K, Baumgart HH, Friedenberg P. Thromboembolism complications in neuro- and thymoleptic therapy [in German]. Dtsch Med Wochenschr. 1967;92:2174-8.

9. Scholz V. Concerning the thromboembolic complications from neuroleptic drugs [in German]. Nervenarzt. 1967;38:174-7.

10. Lal S, Bleiman M, Brown GN. Pulmonary embolism in psychiatric patients. J Am Geriatr Soc. 1966;14:1138-43.

11. Walker AM, Lanza LL, Arellano F, Rothman KJ. Mortality in current and former users of clozapine. Epidemiology. 1997;8:671-7.

12. Hägg S, Spigset $O$, Söderström TG. Association of venous thromboembolism and clozapine. Lancet. 2000;355:1155-6.

13. Zornberg GL, Jick H. Antipsychotic drug use and risk of firsttime idiopathic venous thromboembolism: a case-control study. Lancet. 2000;356:1219-23.

14. Hägg S, Spigset O. Antipsychotic-induced venous thromboembolism: a review of the evidence. CNS Drugs. 2002;16:765-76.

15. Hägg $S$, Jönsson AK, Spigset O. Risk of venous thromboembolism due to antipsychotic drug therapy. Expert Opin Drug Saf. 2009;8:537-47.

16. Jönsson AK, Spigset O, Hägg S. Venous thromboembolism in recipients of antipsychotics: incidence, mechanisms and management. CNS Drugs. 2012;26:649-62.

17. Paciullo CA. Evaluating the association between clozapine and venous thromboembolism. Am J Health Syst Pharm. 2008;65:1825-9.

18. Malý R, Masopust J, Hosák L, Konupcíková K. Assessment of risk of venous thromboembolism and its possible prevention in psychiatric patients. Psychiatry Clin Neurosci. 2008;62:3-8.

19. Neste V, Verbruggen W, Leysen M. Deep venous thrombosis and pulmonary embolism in psychiatric settings. Eur J Psychiatry. 2009;23:19-30.

20. Tromeur C, Couturaud F. Antipsychotic drugs and venous thromboembolism. Thromb Res. 2012;130(Suppl 1):S29-31.

21. Masopust J, Malý R, Vališ M. Risk of venous thromboembolism during treatment with antipsychotic agents. Psychiatry Clin Neurosci. 2012;66:541-52.
22. Zhang R, Dong L, Shao F, Tan X, Ying K. Antipsychotics and venous thromboembolism risk: a meta-analysis. Pharmacopsychiatry. 2011;44:183-8.

23. Barbui C, Conti V, Cipriani A. Antipsychotic drug exposure and risk of venous thromboembolism: a systematic review and metaanalysis of observational studies. Drug Saf. 2014;37:79-90.

24. Brehmer G, Ruckdeschel KT. Technic of the hibernation therapy. Dtsch Med Wochenschr. 1953(78):1724-5.

25. Labhardt F. Technic, side effects and complications of largactil therapy [in German. Schweiz Arch Neurol Psychiatr. 1954;73:338-45.

26. Lunel G, Noble Y, Arné L. The thrombogenic role of neuroleptic agents (apropos of 2 cases) [in French]. Bord Med. 1972;5:121-6.

27. Varia I, Krishnan RR, Davidson J. Deep-vein thrombosis with antipsychotic drugs. Psychosomatics. 1983;24:1097-8.

28. Ruh-Bernhardt D, Finance F, Rohmer F, Singer L. Influence of psychotropic therapy on thrombogenesis and platelet functions. A propos of 4 cases of thromboembolic accidents occurring in patients treated by neuroleptics and antidepressants [in French]. L'Encephale. 1976;2:239-55.

29. Singer L, Finance MF, Ruh MD. Pulmonary embolisms occurring in 1 month in 3 aged women with manic-depressive psychoses. Discussion. Etiopathogenic role or psychiatric treatment [in French]. Ann Med Psychol (Paris). 1975;1:256-63.

30. Mahmoodian MH. Ursachen der Lungenembolie bei psychisch und neurologisch Kranken. Arch Für Psychiatr Nervenkrankh. 1963;204:229-44.

31. Kendel K, Fodor S. Pulmonary embolism and symptomatic psychosis. Ger Med Mon. 1969;14:184-7.

32. Ziegler H. Lungenembolie aus der Sicht des Pathologen. Med Klin. 1977;72:1063-70.

33. Lacut K, Le Gal G, Couturaud F, Cornily G, Leroyer C, Mottier $\mathrm{D}$, et al. Association between antipsychotic drugs, antidepressant drugs and venous thromboembolism: results from the EDITH case-control study. Fundam Clin Pharmacol. 2007;21:643-50.

34. Masopust J, Malý R, Urban A, Hosák L, Čermáková E. Antipsychotic drugs as a risk factor for venous thromboembolism. Int J Psychiatry Clin Pract. 2007;11:246-9.

35. Jönsson AK, Horváth-Puhó E, Hägg S, Pedersen L, Sørensen HT. Antipsychotics and risk of venous thromboembolism: a population-based case-control study. Clin Epidemiol. 2009;1:19-26.

36. Parker C, Coupland C, Hippisley-Cox J. Antipsychotic drugs and risk of venous thromboembolism: nested case-control study. BMJ. 2010;341:c4245.

37. Ishiguro $\mathrm{C}$, Wang $\mathrm{X}, \mathrm{Li} \mathrm{L}$, Jick $\mathrm{S}$. Antipsychotic drugs and risk of idiopathic venous thromboembolism: a nested case-control study using the CPRD. Pharmacoepidemiol Drug Saf. 2014;23:1168-75.

38. Wu C-S, Lin C-C, Chang C-M, Wu K-Y, Liang H-Y, Huang $\mathrm{Y}-\mathrm{W}$, et al. Antipsychotic treatment and the occurrence of venous thromboembolism: a 10-year nationwide registry study. J Clin Psychiatry. 2013;74:918-24.

39. Wang M-T, Liou J-T, Huang Y-W, Lin CW, Wu G-J, Chu C-L, et al. Use of antipsychotics and risk of venous thromboembolism in postmenopausal women. A population-based nested casecontrol study. Thromb Haemost. 2016;115:1209-19.

40. Conti V, Venegoni M, Cocci A, Fortino I, Lora A, Barbui C. Antipsychotic drug exposure and risk of pulmonary embolism: a population-based, nested case-control study. BMC Psychiatry. 2015;15:92.

41. Premuš Marušič A, Petrovič D, Mrhar A, Locatelli I. Polypharmacotherapy and blood products as risk factors for venous thromboembolism in postsurgical patients: a case-control study. Int J Clin Pharm. 2017;39:416-23.

42. Kleijer BC, Heerdink ER, Egberts TCG, Jansen PAF, van Marum RJ. Antipsychotic drug use and the risk of venous thromboembolism in elderly patients. J Clin Psychopharmacol. 2010;30:526-30. 
43. Schmedt N, Garbe E. Antipsychotic drug use and the risk of venous thromboembolism in elderly patients with dementia. J Clin Psychopharmacol. 2013;33:753-8.

44. Parkin L, Skegg DC, Herbison GP, Paul C. Psychotropic drugs and fatal pulmonary embolism. Pharmacoepidemiol Drug Saf. 2003;12:647-52.

45. Hamanaka S, Kamijo Y, Nagai T, Kurihara K, Tanaka K, Soma $\mathrm{K}$, et al. Massive pulmonary thromboembolism demonstrated at necropsy in Japanese psychiatric patients treated with neuroleptics including atypical antipsychotics. Circ J. 2004;68:850-2.

46. Allenet B, Schmidlin S, Genty C, Bosson J-L. Antipsychotic drugs and risk of pulmonary embolism. Pharmacoepidemiol Drug Saf. 2012;21:42-8.

47. Hsu W-Y, Lane H-Y, Lin C-L, Kao C-H. A population-based cohort study on deep vein thrombosis and pulmonary embolism among schizophrenia patients. Schizophr Res. 2015;162:248-52.

48. Ferraris A, Szmulewicz AG, Vazquez FJ, Vollmer WM, Angriman F. Antipsychotic use among adult outpatients and venous thromboembolic disease: a retrospective cohort study. J Clin Psychopharmacol. 2017;37:405-11.

49. Hippisley-Cox J, Coupland C. Development and validation of risk prediction algorithm (QThrombosis) to estimate future risk of venous thromboembolism: prospective cohort study. BMJ. 2011;343:d4656.

50. Nakamura M, Yamada N, Oda E, Matsubayashi D, Ota K, Kobayashi M, et al. Predictors of venous thromboembolism recurrence and the bleeding events identified using a Japanese healthcare database. J Cardiol. 2017;70:155-62.

51. Ray JG, Mamdani MM, Yeo EL. Antipsychotic and antidepressant drug use in the elderly and the risk of venous thromboembolism. Thromb Haemost. 2002;88:205-9.

52. Liperoti R, Pedone C, Lapane KL, Mor V, Bernabei R, Gambassi G. Venous thromboembolism among elderly patients treated with atypical and conventional antipsychotic agents. Arch Intern Med. 2005; 165:2677-82.

53. Dennis M, Shine L, John A, Marchant A, McGregor J, Lyons RA, et al. Risk of adverse outcomes for older people with dementia prescribed antipsychotic medication: a population based e-cohort study. Neurol Ther. 2017;6:57-77.

54. Jönsson AK, Brudin L, Ahlner J, Hedenmalm K, Eriksson A, Hägg S. Antipsychotics associated with pulmonary embolism in a Swedish medicolegal autopsy series. Int Clin Psychopharmacol. 2008;23:263-8.

55. Hägg S, Bate A, Stahl M, Spigset O. Associations between venous thromboembolism and antipsychotics. A study of the WHO database of adverse drug reactions. Drug Saf. 2008;31:685-94.

56. Letmaier M, Grohmann R, Kren C, Toto S, Bleich S, Engel R, et al. Venous thromboembolism during treatment with antipsychotics: results of a drug surveillance programme. World J Biol Psychiatry. 2017;28:1-12.

57. Thomassen R, Vandenbroucke JP, Rosendaal FR. Antipsychotic medication and venous thrombosis. Br J Psychiatry J Ment Sci. 2001;179:63-6.

58. Wolstein J, Grohmann R, Rüther E, Hippius H. Antipsychotic drugs and venous thromboembolism. Lancet. 2000;356:252.

59. Rosendaal FR. Venous thrombosis: the role of genes, environment, and behavior. Hematol Am Soc Hematol Educ Program. 2005;2005:1-12.

60. Huerta C, Johansson S, Wallander M-A, García Rodríguez LA. Risk factors and short-term mortality of venous thromboembolism diagnosed in the primary care setting in the United Kingdom. Arch Intern Med. 2007;167:935-43.

61. Wallaschofski H, Donné M, Eigenthaler M, Hentschel B, Faber $\mathrm{R}$, Stepan H, et al. PRL as a novel potent cofactor for platelet aggregation. J Clin Endocrinol Metab. 2001;86:5912-9.
62. Wallaschofski H, Eigenthaler M, Kiefer M, Donné M, Hentschel B, Gertz HJ, et al. Hyperprolactinemia in patients on antipsychotic drugs causes ADP-stimulated platelet activation that might explain the increased risk for venous thromboembolism: pilot study. J Clin Psychopharmacol. 2003;23:479-83.

63. Urban A, Masopust J, Malý R, Hosák L, Kalnická D. Prolactin as a factor for increased platelet aggregation. Neuro Endocrinol Lett. 2007;28:518-23.

64. Peuskens J, Pani L, Detraux J, De Hert M. The effects of novel and newly approved antipsychotics on serum prolactin levels: a comprehensive review. CNS Drugs. 2014;28:421-53.

65. Axelsson S, Hägg S, Eriksson AC, Lindahl TL, Whiss PA. In vitro effects of antipsychotics on human platelet adhesion and aggregation and plasma coagulation. Clin Exp Pharmacol Physiol. 2007;34:775-80.

66. De Clerck F, Somers Y, Mannaert E, Greenspan A, Eerdekens M. In vitro effects of risperidone and 9-hydroxy-risperidone on human platelet function, plasma coagulation, and fibrinolysis. Clin Ther. 2004;26:1261-73.

67. Schwartz M, Rochas M, Toubi E, Sharf B. The presence of lupus anticoagulant and anticardiolipin antibodies in patients undergoing long-term neuroleptic treatment. J Psychiatry Neurosci. 1999;24:351-2.

68. Lillicrap MS, Wright G, Jones AC. Symptomatic antiphospholipid syndrome induced by chlorpromazine. Br J Rheumatol. 1998;37:346-7.

69. Ayuso J, Saiz Ruiz J. Neuroleptic-induced antinuclear antibodies. Eur Psychiatry. 1996;11:378-9.

70. Canoso RT, de Oliveira RM, Nixon RA. Neuroleptic-associated autoantibodies. A prevalence study. Biol Psychiatry. 1990;27:863-70.

71. Canoso RT, de Oliveira RM. Chlorpromazine-induced anticardiolipin antibodies and lupus anticoagulant: absence of thrombosis. Am J Hematol. 1988;27:272-5.

72. Metzer WS, Canoso RT, Newton JE. Anticardiolipin antibodies in a sample of chronic schizophrenics receiving neuroleptic therapy. South Med J. 1994;87:190-2.

73. Steen VD, Ramsey-Goldman R. Phenothiazine-induced systemic lupus erythematosus with superior vena cava syndrome: case report and review of the literature. Arthritis Rheum. 1988;31:923-6.

74. el-Mallakh RS, Donaldson JO, Kranzler HR, Racy A. Phenothiazine-associated lupus anticoagulant and thrombotic disease. Psychosomatics. 1988;29:109-13.

75. Davis S, Kern HB, Asokan R. Antiphospholipid antibodies associated with clozapine treatment. Am J Hematol. 1994;46:166-7.

76. Shen H, Li R, Xiao H, Zhou Q, Cui Q, Chen J. Higher serum clozapine level is associated with increased antiphospholipid antibodies in schizophrenia patients. J Psychiatr Res. 2009;43:615-9.

77. Roche-Bayard P, Rossi R, Mann JM, Cordier JF, Delahaye JP. Left pulmonary artery thrombosis in chlorpromazine-induced lupus. Chest. 1990;98:1545.

78. Chang S-H, Chiang S-Y, Chiu C-C, Tsai C-C, Tsai H-H, Huang $\mathrm{C}-\mathrm{Y}$, et al. Expression of anti-cardiolipin antibodies and inflammatory associated factors in patients with schizophrenia. Psychiatry Res. 2011;187:341-6.

79. Halacheva K, Dimova S, Tolev T, Dimov D, Nikolova M. Elevated anticardiolipin antibodies in schizophrenic patients before and during neuroleptic medication. Psychiatry Res. 2009;169:51-5.

80. Stubbs B, Williams J, Gaughran F, Craig T. How sedentary are people with psychosis? A systematic review and meta-analysis. Schizophr Res. 2016;171:103-9.

81. McCall WV, Mann SC, Shelp FE, Caroff SN. Fatal pulmonary embolism in the catatonic syndrome: two case reports and a literature review. J Clin Psychiatry. 1995;56:21-5. 
82. Sukov RJ. Thrombophlebitis as a complication of severe catatonia. JAMA. 1972;220:587-8.

83. Ishida T, Sakurai H, Watanabe K, Iwashita S, Mimura M, Uchida $\mathrm{H}$. Incidence of deep vein thrombosis in catatonic patients: a chart review. Psychiatry Res. 2016;241:61-5.

84. Dickerson F, Stallings C, Origoni A, Vaughan C, Khushalani S, Yang S, et al. C-reactive protein is elevated in schizophrenia. Schizophr Res. 2013;143:198-202.

85. Štuhec M. Clozapine-induced elevated C-reactive protein and fever mimic infection. Gen Hosp Psychiatry. 2013;35(680):e5-6.

86. Diaz FJ, Pérez-Iglesias R, Mata I, Martínez-Garcia O, VázquezBarquero JL, de Leon J, et al. Possible effects of some antipsychotic drugs on C-reactive protein in a drug-naïve psychotic sample. Schizophr Res. 2010;121:207-12.

87. Masopust J, Malý R, Andrýs C, Vališ M, Bažant J, Hosák L. Markers of thrombogenesis are activated in unmedicated patients with acute psychosis: a matched case control study. BMC Psychiatry. 2011;11:2.

88. Eldibany MM, Caprini JA. Hyperhomocysteinemia and thrombosis: an overview. Arch Pathol Lab Med. 2007;131:872-84.

89. Reif A, Schneider MF, Kamolz S, Pfuhlmann B. Homocysteinemia in psychiatric disorders: association with dementia and depression, but not schizophrenia in female patients. J Neural Transm (Vienna). 2003;110:1401-11.

90. Cattaneo M. Hyperhomocysteinemia and venous thromboembolism. Semin Thromb Hemost. 2006;32:716-23.

91. National Institute for Health and Care Excellence. Venous thromboembolism in adults: diagnosis and management. NICE guidance and guidelines. https://www.nice.org.uk/guidance/qs29. Cited 14 July 2017.
92. Anderson FA, Wheeler HB, Goldberg RJ, Hosmer DW, Forcier A, Patwardhan NA. Changing clinical practice. Prospective study of the impact of continuing medical education and quality assurance programs on use of prophylaxis for venous thromboembolism. Arch Intern Med. 1994;154:669-77.

93. Durieux P, Nizard R, Ravaud P, Mounier N, Lepage E. A clinical decision support system for prevention of venous thromboembolism: effect on physician behavior. JAMA. 2000;283:2816-21.

94. Al-Dorzi HM, Cherfan A, Al-Harbi S, Al-Askar A, Al-Azzam S, Hroub A, et al. Knowledge of thromboprophylaxis guidelines pre- and post-didactic lectures during a venous thromboembolism awareness day at a tertiary-care hospital. Ann Thorac Med. 2013;8:165-9.

95. Huang W, Anderson FA, Spencer FA, Gallus A, Goldberg RJ. Risk-assessment models for predicting venous thromboembolism among hospitalized non-surgical patients: a systematic review. J Thromb Thrombolysis. 2013;35:67-80.

96. Ye F, Stalvey C, Khuddus MA, Winchester DE, Toklu HZ, Mazza JJ, et al. A systematic review of mobility/immobility in thromboembolism risk assessment models for hospitalized patients. J Thromb Thrombolysis. 2017;44:94-103.

97. Cohen AT, Alikhan R, Arcelus JI, Bergmann J-F, Haas S, Merli GJ, et al. Assessment of venous thromboembolism risk and the benefits of thromboprophylaxis in medical patients. Thromb Haemost. 2005;94:750-9.

98. Liu X, O'Rourke F, Van Nguyen H. Venous thromboembolism in psychogeriatric in-patients-a study of risk assessment, incidence, and current prophylaxis prescribing. Int Psychogeriatr. 2013;25:913-7. 